bowel being gangrenous; the third recovered. One case of ununited fracture of the thigh; ends of bones sawn off and fastened together with strong wire: still under treatment. One resection of elbuw : good result.

\section{CLINICAL LECTURE ON INJURIES OF THE HEAD.}

BY MR. PAGET, F.R.S.

THE subject (on Wednesday last) was illustrated by two cases of injury to the head which have lately been under Mr. Paget's care.

In the first case, a boy, aged II years, met with an accident which caused a compound, slightly depressed, and starred fracture of his left parietal bone. He was stunned for some hours, then vomited, and the next day sat up in bed and answered questions. He had, then, no symptoms of compression, and for that reason he was not trephined. He went on well for twelve days, but, on the thirteenth, he was found to be flushed, and to have a rapid pulse, quick breathing, and a temperature of 103.2; two days later, he was found to have signs of double pneumonia, after which, he gradually sank, and died two months after the accident. At the post mortem examination, both pleuræ were found filled with purulent fluid. The dura mater corresponding to the depressed portion of the bone was inflamed and adherent to the bone ; there was no inflammation of the arachnoid, but, in the substance of the hemisphere immediately beneath the depressed fragment of bone, was a small abscess or slough of brain-substance, about as large as a sixpence in area.

In alluding to this case, Mr. Paget remarked that compound fractures of the skull in young persons are usually recovered from without interference when there are no signs of compression, but considered it possible that in this patient, trephining, although contrary to his usual practice in such cases, might have been beneficial. Mr. Paget thought, however, that it was very doubtful whether the elevation of the depressed fragment would have put the lad in any better position with regard to pyæmia than leaving it alone. In referring to the treatment of the secondary inflammation in cases like this, Mr. Paget said that he was not aware of any rule which could be laid down on the subject secondary trephining in the present case would clearly have been absurd.

On the question of trephining for injuries of the head, Mr. Paget said he did not consider that large enough statistics of successes and failures had yet been collected to admit of our forming rules which shall be constantly applicable. Mr. Paget gave the following provisional laws as, in his opinion, expressing the results of our present knowledge.

r. Simple Fractures of the Skull. - a. When there are no signs of compression, and no depression, leave the patient alone. $b$. Without depression, but with signs of compression (i.e., abiding insensibility), we may interfere if we can diagnose the seat of fracture. $c$. When there is depression, but no sign of compression, it is best to do nothing if the patient be young; if old, however, there is some doubt about the pro. priety of non-interference. $d$. When there is depression and compression, we must operate.

2. Compound Fractures. - a. When there is no depression, and no sign of compression, as a rule-especially in young patients-do nothing. $b$. When there is no depression, but there are signs of compression, it is right to interfere. $c$. With depression, but no signs of compression, it is best not to interfere when the patient is young; but probably it is right to operate on an old person. $d$. With depression and abiding insensibility, operate.

The second patient to whom Mr. Paget referred was a man who was admitted for compound fracture of the lower jaw and of the nasal bones, lacerations, and contusions. He was supposed, from appearance after the accident, to be drunk. The next day he was quite sensible, but restless; on the fourth or fifth day, he became partly comatose; his breathing was very quick, and his face became much swollen and erysipelatous. Mr. Paget diagnosed some secondary erysipelatous inflammation in the cranium. The man continued to get worse, and died. At the post mortem examination, a fracture was found extending through the nasal bones, superior and inferior maxillæ, ethmoid, sphenoid and basilar process of the occipital bone, into the foramen magnum. Puro. lymph in abundance lined the dura mater, and pus was found in the meshes of the pia mater, especially along the lines of the larger arteries. Mr. Paget called attention to the disproportion between the immediate symptoms in this case and the extent of the injury, and remarked that fractures of the base are frequently unattended by signs of compression. Mr. Paget also noticed, in this connection, that fractures of the anterior part of the skull are far less fatal than those of the posterior part.
Mr. Paget alluded, in connection with these two cases, to the nature of the secondary inflammation, and insisted on the importance of drawing a broad line in practice, between an inflammation which results directly from an injury and one which follows it at an interval of several days; the former being sthenic, the latter asthenic and allied to the erysipelatous inflammations.

This led to the subject of erysipelas. Mr. Paget referred to a case now under his care, which illustrated the tendency of erysipelas to shift about from one part or organ to another. The disease, Mr. Paget remarked, is well known to shift from one part of the surface to another, and from the surface to a deeper organ, but it very rarely shifts from a deep structure to one more superficial. Mr. Paget mentioned the following case as an illustration of this rare condition. The patient (a little girl) was admitted on account of a severe burn. She became affected with delirium, fever, and restlessness, and these symptoms led Mr. Paget to diagnose an inflammation of the brain-membranes. When she had been in this state for three days, however, erysipelas appeared on the scalp, and, simultaneously, the delirium was relieved, showing, in $\mathrm{Mr}$. Paget's opinion, a translation of the erysipelatous inflammation from her brain membranes to her scalp.

In connection with the recent occurrence of some cases of erysipelas in his wards, Mr. Paget made some remarks on the extreme importance of cleanliness in the treatment of this disease, especially with the view of preventing infection. Mr. Paget said that, although several cases of erysipelas had been in the neighbourhood of patients on whom operations had lately been performed, and had even originated in the wards, yet no single case of propagation of the disease had occurred; and, that he believed this result to be mainly due to scrupulous care in keeping everything about the patients and in the wards very clean, dry, and well ventilated.

\section{REVIEWS AND NOTICES.}

Sanitary Works and Sewage Utilisation. By J. Bailey DenTON, M.Inst.C.E. - REPORT OF THE BRITISH ASSOCIATION COMMitTeE ON THE TReatMent and UTilisation OF Sewage. Drawn up by Dr. B. H. PAUL, at the request of the Committee.The Distribution and Agricultural Use of Town Sewage. By W. HOPE, V.C.; a Paper read at the Institution of Surveyors. THE subject treated of in these three pamphlets is one that has long been a source of vexation, and for that reason there is too often a tendeency to avoid it ; but this subject is so intimately connected with the comfort and health of a civilised community, and its disregard is capable of giving rise to such serious consequences, that it cannot long be neglected with impunity. It must, before long; take a prominent place before the public and the Government; and those who are concerned in its being suitably dealt with, will need to consider thoroughly all the circumstances of the case. Mr. BAILEY Denton's pamphlet furnishes an idea of some few of the perplexities and deficiencies experienced in the attempt to introduce sanitary improvements, and it indicates some of the difficulties which either exist already or will have to be dealt with hereafter.

The preliminary Report of the British Association Committee professes merely to give a statement of the present position of the subject. After reviewing the history of the measures adopted for cleansing towns and improving their sanitary condition, by removing the refuse of their inhabitants, up to the time when the general adoption of water as the means of effecting those objects gave rise to the difficulties now prevailing, the conclusions come to by the Sewage Commissioners between 1857 and 1865 are quoted, for the purpose of showing that there is no longer any question as to what is the right way to dispose of liquid town-sewage ; that its judicious ?application to land is profitable, and that the disposal of sewage in this way is the only means of preventing that pollution of rivers which has become a national nuisance.

But those conclusions do not by any means exhaust the subject. It still remains to be considered how the work to which they point is to be done ; moreover, in regard to small places, it is still a question whe. ther the water-carriage system of removing house-refuse is the most appropriate.* The dry-earth system has, at least, many advocates; and its applicability, as compared with the water-system, has never yet met with thorough impartial investigation. In the midland counties, again, modifications of the most primitive mode of dealing with human excreta are decidedly in favour, and they are preferred to the water-carriage system of removal, on the grounds that they are more advantageous in every way. On the Continent, too, there appears to be a prevailing

* In a pamphlet lately published by the Rev. Mr. Moule. 